\title{
Detection of Fake News using Machine Learning
}

\author{
Prof.Shivaprasad More ${ }^{1}$, Aditya Jadhav ${ }^{2}, S_{\text {Shubham } \operatorname{Yadav}^{3}, \text { Sakshi Chavan }^{4} \text {,Neha More }}{ }^{5}$ \\ Assistant Professor,Department of Computer Science and Engineering,Sanjay Ghodawat University,Kolhapur,India ${ }^{1}$
}

Research Scholars,Department of Computer Science and Engineering,Sanjay Ghodawat University,Kolhapur,India ${ }^{2,3,4,5}$

\begin{abstract}
Information is very important today,every time we read a piece of information or watch news on TV,we look for reliable source.Nowadays fake news is making various issues from harsh articles to made news and planning government proclamations in specific outlets.Fake news and nonattendance of trust in the media are creating issues with enormous ramifications in our overall public.Obviously,a deliberately deceptive story is "fake news" yet recently babbling online media talk is changing its definition[1].Some of them currently utilize the term to excuse the realities counter to their favored perspectives.In this today's era we try to get an information or news from the internet and from the social media handles or YouTube,but Information quality in social media is an increasingly important issue.There are so many fake news or tampered video news spread all over the internet and social media.A large portion of the fake news identification frameworks use the semantic element of the news. Nonetheless,they experience issues in detecting profoundly equivocal fraudulent news which can be recognized solely after distinguishing its importance and most recent related data. Therefore,identifying whether the news in text as well as in video format is fake or real has become an important aspect.So,we intended to solve this problem by using different techniques and algorithms. We will be proposing a system where it will be very easy to identify whether the given NEWS on Social Media is Real or Fake,this NEWS can be in Text form[2].
\end{abstract}

Keywords: Fake,Real,Social Media,News,Algorithms,Machine Learning

\section{INTRODUCTION}

Information is very important today,every time we read a piece of information or watch news on TV,we look for reliable source.Nowadays fake news is making various issues from harsh articles to made news and planning government proclamations in specific outlets.Fake news and non attendance of trust in the media are creating issues with enormous ramifications in our overall public.Obviously,a deliberately deceptive story is "fake news" yet recently babbling online media talk is changing its definition[1]. Some of them currently utilize the term to excuse the realities counter to their favored perspectives.In this today's era we try to get an information or news from the internet and from the social media handles or YouTube,but Information quality in social media is an increasingly important issue. There are so many fake news or tampered video news spread all over the internet and social media.A large portion of the false news identification frameworks use the semantic element of the news. Nonetheless,they experience issues in detecting profoundly equivocal fraudulent news which can be recognized solely after distinguishing its importance and most recent related data. Therefore,identifying whether the news in text as well as in video format is fake or real has become an important aspect. So,we intended to solve this problem by using different techniques and algorithms. We will be proposing a system where it will be very easy to identify whether the given NEWS on Social Media is Real or Fake,this NEWS can be in Text form[3].

\section{LITERATURE REVIEW}

International Network is one of the significant creations and an enormous number of people are its clients.People on earth utilize this for various purposes. There are assorted online media stages that are available to these customers.Any customer can make a post or spread any sort of information through the online stages. These destinations or applications or stages don't approve the customers or their posts.So some of the customers endeavor to spread fake news through these stages.These news can be intentional exposure against an individual,society,affiliation or philosophical gathering.An individual can't distinguish all these fake news So there is a requirement for AI[4] classifiers that can recognize these fake news consequently.

\section{II.A. The Impact of Fake News}

The internet is essentially determined by commercials .Websites with thrilling features are famous, which prompts promoting organizations gaining by the high traffic to the site.It was along these lines found that the designers of fake news sites and data could bring in cash through mechanized publicizing that rewards high traffic to their websites.The question remains how falsehood would then impact general society[5]. The spreading of wrong information can create 


\section{International Journal of Advanced Research in Computer and Communication Engineering}

Vol. 10, Issue 4, April 2021

DOI 10.17148/IJARCCE.2021.10484

turmoil and superfluous pressure among the public.Fake news that has deliberately made to deceive and have hurt the general population is alluded to as advanced disinformation.Disinformation can probably cause issues, in practically no time,for a huge number of individuals. Disinformation has been known to disturb political decision measures,make disquiet,debates and aggression among people in general[6].

\section{II.B. Fake News and Social Media}

Nowadays,the web has become an essential piece of our everyday lives .Traditional techniques for getting data have almost disappeared to make ready for web-based media stages.It was represented in 2017 that Facebook was the greatest electronic media stage,working with more 1.9 million customers around the world.The part of Facebook in the spreading of fake news perhaps has the greatest effect from all the online media platforms.It was accounted for that $44 \%$ of overall clients get their report from Facebook.23\% of Facebook clients have demonstrated that they have shared bogus data,either purposely or not.The spreading of false news is powered by online media stages and it is occurring at a disturbing speed[7].

\section{II.C. Findings}

While leading the writing review, 22 articles included the social segments; 13 articles discussed the work that scholarly factors have in adding to the sharing and spreading of fake news; 13 articles highlighted the piece of political components;nine articles analyzed how money related advantage could convince an online media customers to spread false information and 13 articles talked about noxious components and the effect that malignant components have on the sharing and spreading of fake information.Figure 2.1 gives a breakdown of each of the 38 articles containing references to every one of the sub-classifications recorded previously.It was obviously apparent that the two single subclasses of social correlation and disdain publicity were the most discussed.With the subfactor,information and training,intently behind.A high level of the articles,34.2\% (13 of 38),allude with the impacts of social examination on the spreading of bogus data;trailed by $26.3 \%$ (10 of 38) of the articles referring to despise purposeful publicity.Information and schooling was estimated at $23.6 \%$ ( 9 of 38 ).

\section{Sub-Categories of the Spreading of Fake News}

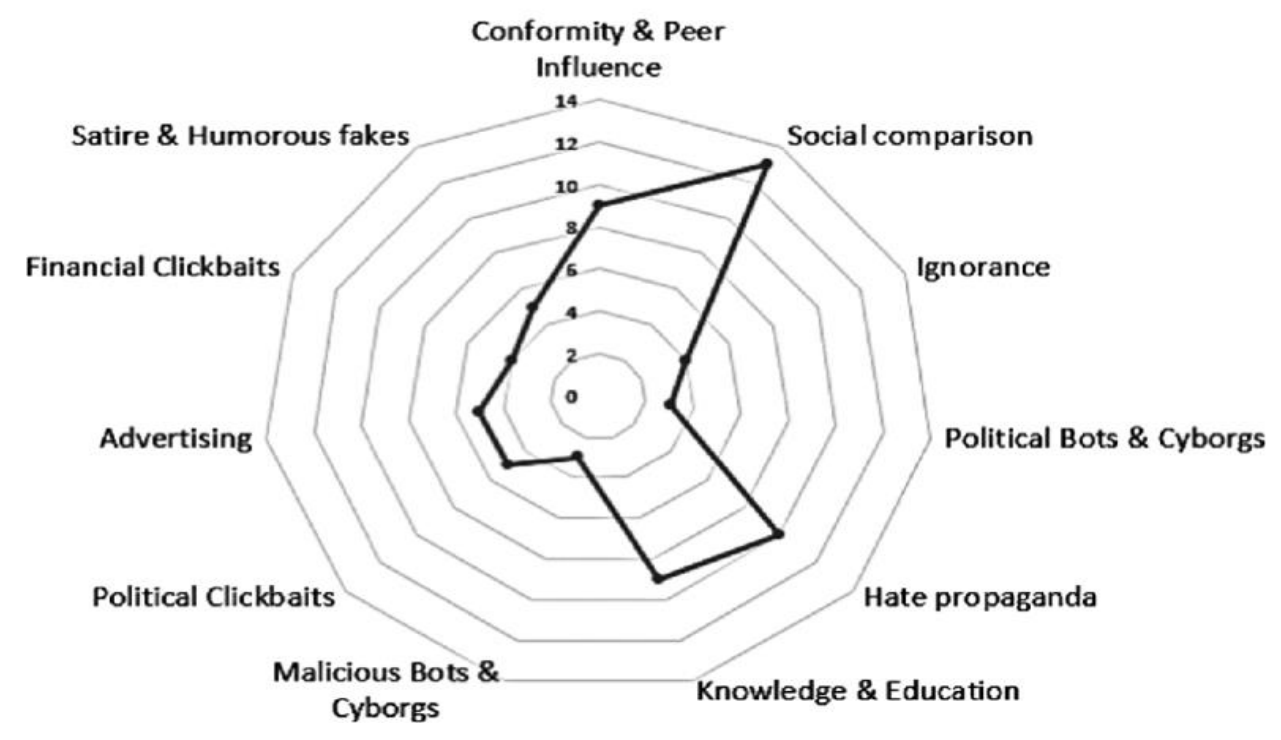

Fig .1 Articles discussing the sub-categories of the spreading of fake news

Moreover,it was deduced that the vast majority of the 38 articles included a mix of the social factors for instance congruity and partner sway,social relationship and spoof and humorous fakes,which measures at 60.5\% (23 of 38). Where the mix of the mental components for instance data and tutoring and neglectfulness assessed at $39.4 \%$ (15 of 38).Political factors and sub-factors for instance political misdirecting content sources and political bots/cyborgs,were discussed in $34.2 \%$ (13 of 38) of the articles.Besides,money related factors for instance publicizing and financial deluding content sources were alluded to in $23.6 \%$ (9 of 38) of the journal articles.Furthermore,finally,pernicious elements for example noxious bots and cyborgs,disdain promulgation and malignant misleading content sources estimated at $34.2 \%$ (13 of 38 ). 


\section{METHODOLOGY}

- Data set is loaded and Exploratory Data Analysis or (EDA) is done on that data set.

- After that the prepossessing is done which includes:

1. All words except 52 words other letters are eliminated[13].

2. Then,Converts all upper case to lower case.

3. Then,the lower case alphabets/letter are split into single word from sentence.

4. After that,the stop word are fetched (i.e. common words).

5. The,it combines all stemmed words and convert it into a sentence.

6. At last,all pre-processed words are stored in review to display.

- $\quad$ Pre-process and transform data point text and try to predict it based on the model:

1. It takes input from the repriced data.

2. After that,Using TF-IDF Vectorizer it transfer the data into array and after that it convert words into meaning full no. that is 0 and 1 .

3. If word is has produced the result as 0 it is 'Fake',else it is 'Real'.

- $\quad$ Saving model and TF-IDF Vectorizer:

1. You can utilize the pickle operation to serialize your AI calculations and save the serialized arrangement in a file.

2. Once the model is saved we can use scikit-learn in our destination application (e.g.,web application) to load the model.

- $\quad$ Load model and Vectorizer and predict on previous pre-processed data point.

- $\quad$ Save some data points for text random generation.

\section{IMPLEMENTATION}

In this project,we have used some of the libraries which are required to produce the accurate result.The libraries are Flask,nltk,Flask_WTF,WTForms,pandas,scikit_learn,gunicorn,joblib.

Flask: Flask is a popular Python web framework,which we have used for deigning the web application for the project. The version used of Flask is Flask==1.1.1.

NLTK:NLTK is a main stage for building Python projects to work with human language information.In our venture we have utilized NLTK for text handling libraries for arrangement,tokenization,temming and labeling parsing,and semantic thinking. The form utilized of nltk is nltk==3.4.5.[7]

Flask_WTF:WTF represents WT Forms which is planned to give the intelligent UI to the client.The WTF is an underlying module of the carafe which gives an elective method of planning structures in the cup web applications. The form utilized of Flask_WTF== 0.14.3.

WTForms:WTForms is an adaptable structures approval and delivering library for Python web improvement.The adaptation utilized of WTForms $==2.3 .1$.

Scikit_learn:[8]The sklearn library contains a great deal of productive instruments for AI and measurable demonstrating including characterization,regression grouping and dimensionality decrease.The version used of scikit_learn==0.23.1.

Pandas: Pandas is an open source Python bundle that is most generally utilized for information science/information examination and $\mathrm{AI}$ assignments. The variant utilized of pandas==0.25.1. 


\section{International Journal of Advanced Research in Computer and Communication Engineering}

Vol. 10, Issue 4, April 2021

DOI 10.17148/IJARCCE.2021.10484

Gunicorn:Green Unicorn,regularly abbreviated to "Gunicorn",is a Web Server Gateway Interface (WSGI) worker execution that is usually used to run Python web applications. The variant utilized of gunicorn==20.0.4 .

Joblib: Joblib is a set of tools to provide lightweight pipe lining in Python.Specifically,joblib offers: straightforward circle reserving of the yield esteems and lethargic re-assessment. The form utilized of joblib= $=0.14 .1$

sklearn.feature_extraction.text.TfidfVectorizer: Convert an collection of crude reports to a matrix of TF-IDF highlights.It transfers the data into array and after words are converted to meaningful number.

The pickle module is used for executing twofold shows for serializing and de-serializing a Python object structure.

1.Pickling: It is a cycle where a Python object progression is changed over into a byte stream

2.Unpickling: It is the backwards of Pickling measure where a byte stream is changed over into an article order.

\section{RESULT AND ANALYSIS}

\section{V.A. Accuracy Comparison of Different models for Fake news Detection}

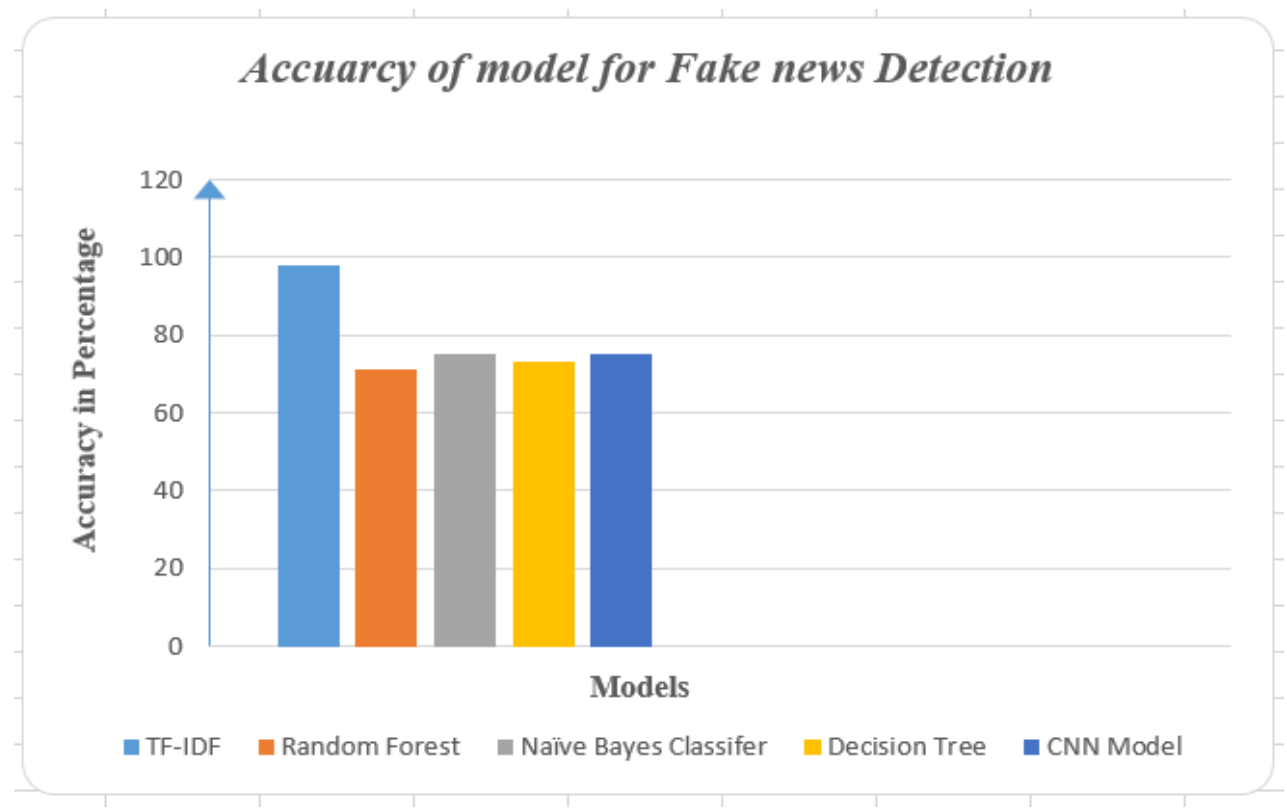

Fig 2. Accuracy of different models

Above Graph displays the accuracy of the system using tf-idf model and eventually the other models in machine learning like Random forest,Naive Bayes Classifier etc B are compared for accuracy.Maximum Accuracy is obtained in tf-idf model due to huge amount of random dataset[9] is giving to the model that helps in predicting news the in accurate way.

For Other Models the accuracy goes on decreasing as the number of words go on increasing as given by user as input to the system.But in our system the random dataset[11] and the NLTK[12] tool with latest version helps in giving the perfect output without considering the number of words in the news and also pickling and unplicking methods boost the predicting the output with perfect accuracy.

\section{V.B. CONFUSION MATRIX}

Below graph shows the confusion matrix for accuracy for the system 
DOI 10.17148/IJARCCE.2021.10484

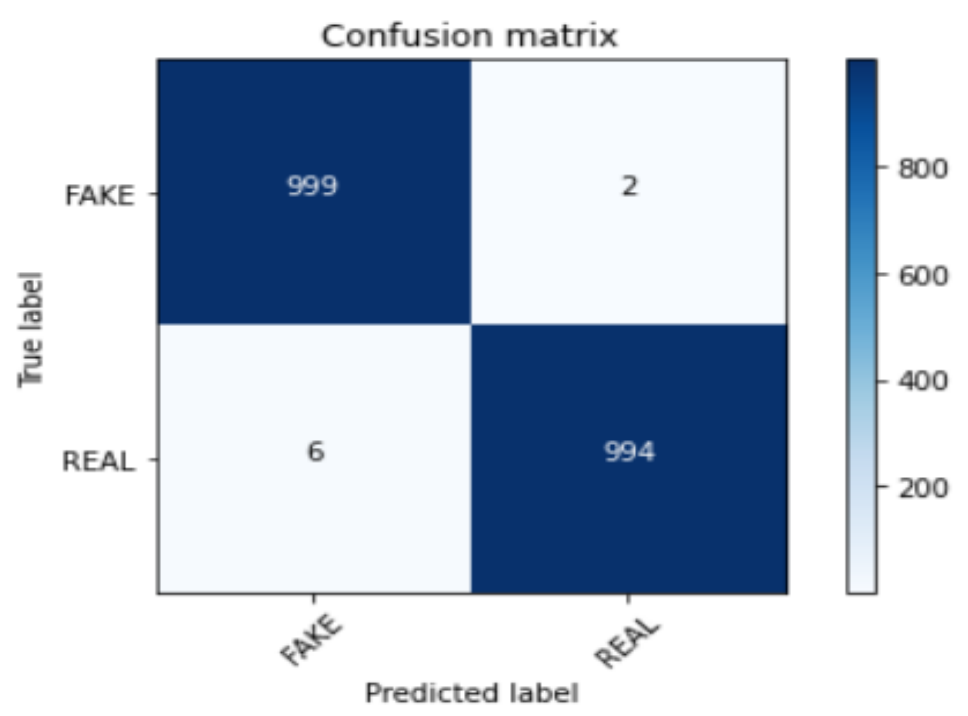

Fig 2. Confusion Matrix

A confusion matrix is a table that is regularly used to portray the introduction of a gathering model (or classifier) on a lot of test data for which the real characteristics are known.It allows the impression of the introduction of an estimation[14].A confusion network is a summary of assumptions coming about on a gathering issue. The amount of right and mistaken estimates are summarized with check regards and isolated by each class. This is the route into the disorder organization. The disorder grid shows the way your portrayal model is perplexed when it makes figures.It gives us understanding not simply into the mix-ups being made by a classifier anyway more fundamentally the sorts of goofs that are being made[10].

[15]Confusion Matrices simpler to look at and read,so I utilized the scikit-learn documentation to fabricate some effectively comprehensible disarray networks.A disarray lattice shows the appropriate marks on the primary inclining (upper left to base right).Different cells show off base names,regularly alluded to as bogus positives or bogus negatives.Contingent upon your concern,one of these may be more significant.

Total Class 1 (Predicted) Class 2 (Predicted)

$\begin{array}{lll}\text { Class } 1 \text { (Actual) } \quad \text { TP } & \text { FN }\end{array}$

$\begin{array}{lll}\text { Class } 2 \text { (Actual) } \quad \text { FP } & \text { TN }\end{array}$

Table 1: Confusion Matrix

\section{VI.FUTURE WORK}

$>\quad$ Detection of Whole Web Pages using NLP

$>\quad$ Deep fake Detection using RNN and LSTM

$>\quad$ Detection of Fraud Websites and Links

$>\quad$ Misinformation Detection

\section{CONCLUSION}

The errand of requesting news genuinely needs start to finish data on the space and inclination to recognize peculiarities in the substance.In this investigation,we analyzed the issue of collection fake reports using AI models and troop strategies. The data we used in our work is accumulated from the World Wide Web and contains reports from 


\section{International Journal of Advanced Research in Computer and Communication Engineering}

Vol. 10, Issue 4, April 2021

\section{DOI 10.17148/IJARCCE.2021.10484}

various spaces to cover an enormous part of the news instead of unequivocally portrayed political news. The learning models were arranged and limit tuned to get ideal exactness.A couple of models have achieved comparably higher exactness than others. We utilized different execution measurements to analyze the outcomes for every calculation.

Fake news identification has many open issues that require the consideration of analysts.For instance, In solicitation to decrease the spread of deceitful news,recognizing key segments drawn in with the spread of data is a huge step.Graph hypothesis and AI procedures can be used to perceive the key sources and draw in with spreads of fake news.

In the 21 st century,Papers that were previously liked as printed copies are currently subbed by applications like Facebook,Twitter, and news stories to be perused on the web. Whatsapp advances are additionally a significant source.The developing issue of false news just makes things more convoluted and attempts to change or hamper the assessment and demeanor of individuals towards utilization of advanced innovation. At the point when an individual is misdirected by the genuine news two potential things happen-People begin to accept that their discernment about a specific subject is valid as expected.Hence,to check the level,we have built up our Fake news Detection framework that takes contributions from the client and orders it to be valid or fake.The model is prepared utilizing a suitable dataset and execution assessment is likewise done utilizing different execution measures.The best model,for example,the model with the most noteworthy exactness is utilized to characterize the news features or articles. The client can check the news story or catchphrases on the web; he can likewise check the validness of the site. The accuracy for dynamic system is $99 \%$ with TF-IDF model and it increases with every iteration. We purpose to assemble our dataset which will stay up with the latest as per the most recent news.All the live news and most recent information will be kept in a data set utilizing Web Crawler and online data set.

\section{REFERENCES}

[1]. Kai Shu,Amy Sliva,Suhang Wang,Jiliang Tang,and Huan Liu,Fake News Detection on Social Media:A Data Mining Perspective arXiv: $1708.01967 \mathrm{v} 3$ [cs.SI],3 Sep 2017

[2]. M. Granik and V. Mesyura,"Fake news detection using naive Bayes classifier," 2017 IEEE First Ukraine Conference on Electrical and Computer Engineering (UKRCON),Kiev,2017,pp. 900-903.

[3]. Fake news websites. (n.d.) Wikipedia. [Online]. Available: https://en.wikipedia.org/wiki/Fake_news_website.Accessed Feb. 6,2017

[4]. Cade Metz. (2016,Dec. 16). The bittersweet sweepstakes to build an AI that destroys fake news.

[5]. Conroy,N.,Rubin,V. and Chen,Y. (2015).Automatic deception detection: Methods for finding fake news at Proceedings of the Association for Information Science and Technology,52(1),pp.1-4.

[6]. Markines,B.,Cattuto,C.,\& Menczer,F. (2009,April). Social spam detection. In Proceedings of the 5th International Workshop on Adversarial Information Retrieval on the Web (pp. 41-48)

[7]. Kushal Agarwalla,Shubham Nandan,Varun Anil Nai,D. Deva Hema,Fake News Detection using Machine Learning and Natural Language Processing,International Journal of Recent Technology and Engineering (IJRTE) ISSN: 2277-3878,Volume-7,Issue-6,March 2019

[8]. Scikit-Learn- Machine Learning In Python

[9]. Dataset- Fake News detection William Yang Wang. " liar,liar pants on _re": A new benchmark dataset for fake news detection. arXiv preprint arXiv: $1705.00648,2017$.

[10]. Aayush Ranjan,Fake News Detection Using Machine Learning,Department Of Computer Science \& Engineering Delhi Technological University,July 2018.

[11]. Patil S.M.,Malik A.K. (2019) Correlation Based Real-Time Data Analysis of Graduate Students Behaviour. In: Santosh K.,Hegadi R. (eds) Recent Trends in Image Processing and Pattern Recognition. RTIP2R 2018. Communications in Computer and Information Science,vol 1037. Springer,Singapore.

[12]. Badreesh Shetty,Natural Language Processing (NLP) for machine learning at towardsdatascience,Medium.

[13]. Ultimate guide to deal with Text Data (using Python) for Data Scientists and Engineers by Shubham Jain,February 27,2018

[14]. Aayush Ranjan,Fake News Detection Using Machine Learning,Department Of Computer Science \& Engineering Delhi Technological University,July 2018.

[15]. What is a Confusion Matrix in Machine Learning by Jason Brownlee on November 18,2016 in Code Algorithms From Scratch. 